\title{
The design and implementation of caloric restriction for oncology research (CaReFOR): Is starving cancer cells in the clinical realm feasible?
}

\author{
Brittany A Simone ${ }^{1}$, Pramila Rani Anne ${ }^{1}$, Kevin Ko ${ }^{1}$, Mak Sarich $^{1}$, Kimberly Strickland ${ }^{1}$, Daniel Monti ${ }^{2}$, Adam C Berger ${ }^{3}$ and Nicole L Simone $^{1}$ \\ ${ }^{1}$ Department of Radiation Oncology, Sidney Kimmel Cancer Center, Jefferson Medical College of Thomas Jefferson University, Philadelphia, PA \\ ${ }^{2}$ Myrna Brynd Center for Integrative Medicine, Thomas Jefferson University Hospital, Philadelphia, PA \\ ${ }^{3}$ Department of Surgery, Kimmel Cancer Center, Jefferson Medical College of Thomas Jefferson University, Philadelphia, PA
}

\begin{abstract}
Metabolic alterations are now known to underly cancer growth and progression and therapeutic options are being designed to target dysregulation of metabolism. Using dietary alterations in the treatment of cancer, would be an idea way to intervene in a non-toxic manner. While nutritional interventions have been previously used in the setting of cancer prevention and survivorship, we propose using diet as a cancer therapeutic. Preclinical data demonstrate that caloric restriction (CR) augments radiation therapy by decreasing growth of tumor cells and increasing overall survival. We have therefore designed and implemented a clinical trial to determine if caloric restriction could be tolerated in the clinic during cancer therapy. The CaRe FOR Study: A Pilot Trial Evaluating Caloric Restriction for Oncology Research in Early Stage Breast Cancer Patients. Patients with early stage breast cancer, who require breast radiation have been enrolled. The patients undergo a 10 week CR program, during which they reduce their baseline calories by $25 \%$ for 2 weeks pre-radiation, 6 weeks during radiation, and 2 weeks post-treatment. The primary endpoint is to determine if breast cancer patients can adhere to CR during radiation therapy. After enrolling, patients log their baseline caloric intake in an online food diary for one week and a calorie target is established by the provider by calculating a 25\% reduction. Patients begin dietary alterations after they receive personalized dietary counseling and behavioral modification lessons adapted from the Diabetes Prevention Program, which both continue through the 10 week program. Secondary parameters will determine if an objective measure can be used to monitor patient adherence in the future and include weight and body composition measures, serum biomarkers, quality of life parameters and treatment toxicity. Here, the steps involved in trying to design a successful clinical trial using CR concurrently with standard cancer treatment such as radiation will be outlined. The long-term goal of the study is to be able to guide methods by which dietary interventions can be successfully implemented for all cancer patients and and to demonstrate that CR is a viable treatment option.. This commentary will provide insight into methods to design clinical trials using dietary alterations.
\end{abstract}

\section{Background}

Metabolic pathways have been implicated in many diseases including cancer. Recently, in the setting of oncology prevention or survivorship, efforts to modify these pathways have been investigated with pharmaceutical agents such as metformin, or with dietary interventions [1]. Mounting preclinical data demonstrates the benefit of using diet modification to enhance the efficacy of cancer treatment. In murine models, caloric restriction (CR) combined with radiation therapy (RT) increases tumor regression and delays metastases [2]. Additional preclinical research demonstrates a significant increase in survival and changes in cancer-related biomarkers with combination therapy. Our current interest lies not only in determining if radiation therapy combined with caloric restriction is clinically feasible, but if it is more effective than standard interventions. The environment of patient care and the stress of cancer treatment can make clinical dietary intervention challenging. Although a dietary intervention may be potentially beneficial to patients, it is uncertain if they will be able to adhere to such a treatment while undergoing cancer therapy. Successful dietary intervention depends on many factors such as a multi-disciplinary approach combining nutritional counseling, and behavioral modification.

The population we chose to begin a dietary intervention during cancer treatments was women with breast cancer. First, preclinical data indicates CR augments standard cytotoxic therapies in this population [2,3]. Second, breast cancer patient's BMI and weight gain directly correlates with outcomes for early and late stage patients [4]. Observational studies show that breast cancer patients often gain weight during the first year of their cancer treatment, with premenopausal women gaining an average of 10 pounds and postmenopausal women gaining an average of 20 pounds $[5,6]$. In addition to having higher rates of breast cancer recurrence and mortality [7], this weight gain is linked to worse outcomes in terms of cancer-related symptoms and quality of life [8]. Third, existing standard therapies, such as anti-estrogen therapies like tamoxifen and anastrazole, and steroids administered as a pre-chemotherapy can often cause altered metabolism and weight gain and an intervention could help behavioral modifications to combat since weight is linked to outcomes in this disease.

Correspondence to: Nicole L. Simone, MD, Bodine Cancer Center, Department of Radiation Oncology, PA 19107, Philadelphia, Tel: (215) 503-0554; E-mail: Nicole.simone@jeffersonhospital.org

Key words: clinical trial, diet, caloric restriction, oncology, breast cancer, radiation Received: September 20, 2017; Accepted: October 06, 2017; Published: October 09,2017 
The CaReFOR (Caloric Restriction For Oncology Research) trial is a pilot study which we have designed to investigate the feasibility of the combination of CR with cancer therapy. This first interventional trial will use dietary manipulation with standard radiation in early stage breast cancer patients. To date, it remains the only trial (per clinicaltrials. gov) to combine CR concurrently with radiation therapy. In this trial, Stage 0 and 1 breast cancer patients undergoing whole breast radiation therapy following lumpectomy are subject to $\mathrm{CR}$ by decreasing their total normal baseline caloric intake by $25 \%$. The primary endpoint of the trial is patient adherence to CR while undergoing radiation therapy. Given that this trial is a first-in-kind, this manuscript details the study design, potential pitfalls and unique aspects of our protocol aimed to achieve $80 \%$ compliance, and to generate reproducible outcomes that can be translated into larger, multi-institutional settings.

\section{Methods}

\section{Design and endpoints}

Mounting data suggest that CR may be used in conjunction with standard cancer therapeutics to increase efficacy and decrease toxicity, but opposition to dietary intervention exists. Critics often cite patient compliance and providers' ability to monitor compliance as potential issues in implementing this intervention. We therefore sought to design a feasibility trial, given that $\mathrm{CR}$ had not been previously used in the setting of a clinical trial with radiation therapy. The CaReFOR was designed to prove the hypothesis that women undergoing whole breast radiation therapy for early stage breast cancer would be able to successfully adhere to CR with a compliance rate of $80 \%$ for all dietary events that they logged. The study is an investigator initiated collaboration between the Radiation Oncology Department and the Integrative Medicine Department at the Sidney Kimmel Medical College of Thomas Jefferson University and was reviewed and approved by the Institutional Review Board (IRB). The study was designed as a single arm trial with no randomization. The primary endpoint was adherence to a $25 \%$ reduction in total caloric intake over a 10 -week period that started prior to radiation therapy and extended until two weeks after completion of radiation treatment. Secondary endpoints include: biometric evaluation, weight loss, patient reported outcomes psychosocial evaluation, physician reported toxicity, and translational endpoints pertaining to serum biomarkers and tumor biology.

Logistical considerations to begin dietary trials are multi-faceted. The design of this trial has been streamlined so procedures can be applied at other institutions. Initial consultation with a physician involves an extensive discussion on the potential benefits, risks and commitment needed to participate in a CR trial. Seven to ten days prior to the patients planning session for radiation (simulation), the patient logs all of their dietary consumption to establish their baseline caloric intake. Then, on the day of the radiation simulation, patients have dietary counseling to discuss their baseline food diaries and tailor an individualized $25 \%$ reduction in calories. Patients begin the CR diet the day after simulation and remain on the diet for the next 10 weeks during which time they are monitored weekly. Patients receive concurrent ionizing radiation (IR) during weeks 3-8 of the diet. The trial and data collection lasts for a total of approximately 18 weeks with one follow up visit occurring 4 weeks after the completion of radiation.

\section{Eligibility criteria}

When designing trials using dietary alterations, unique aspects must be considered in order to ensure success of both the primary endpoint and the translational secondary endpoints. In this first-in-human trial, the primary endpoint was chosen to determine adherence to calorie reduction, enrolled patients must be able to tolerate that reduction without adverse consequences. In addition, secondary translational endpoints to assess the effect CR has on a patient undergoing cancer therapy such as biometric analysis, quality of life studies, and serum biomarkers corresponding to the degree of caloric restriction were included to determine if dietary adherence and success can be evaluated objectively and defined. In this section, some key aspects of eligibility criteria will be discussed and stringent inclusion/exclusion criteria are defined. In brief, the trial was designed for women with a pathologically proven diagnosis of DCIS or invasive ductal carcinoma who are Stage 0 or I, and are able to have breast conservation.

Weight and BMI: Due to the direct impact reduction of calories can have on weight, consideration is given to baseline weight and BMI as eligibility parameters. Previous trials involving CR have typically included patients of normal weight and without significant co-morbidities, it was decided that normal weight, overweight and obese patients alike should be enrolled. The Nurses Health Initiative demonstrates that weight gain during cancer therapy is a poor prognostic factor in breast cancer prognosis and recurrence. Therefore, caloric restriction in obese or overweight populations may be beneficial in a multifactorial manner. In addition, since weight gain is experienced by $50-90 \%$ of breast cancer patients, particularly those patients who are postmenopausal, it was inferred that this would be an appropriate patient demographic. Conversely, since weight loss of up to $15 \%$ of body weight has been associated with CR, we determined that it would be most appropriate to exclude patients with a BMI less than $21 \mathrm{~kg} / \mathrm{m}^{2}$ and those who had experienced weight loss of $10 \%$ or more of their body weight in 3 months prior to enrollment $[9,10]$. As research and trials implementing CR evolve, it can be envisioned that the patient's BMI and other factors may be used to individualize a proper CR protocol for oncologic patients.

Metabolic considerations: As a secondary endpoint of this trial, serum biomarkers are to be assessed to determine a potential way to determine patient adherence but also to ascertain benefit of CR in cancer patients. Therefore, the patients' metabolic state was considered in designing the eligibility portion of this trial. It is anticipated that potential biomarkers corresponding to caloric restriction will be used to assess patient adherence. As a result, considerations have been made to decrease variables which would affect metabolism and perhaps obscure otherwise significant markers. Patients chronically taking steroids are excluded since steroids are known to cause metabolic disturbances and may affect measured serum biomarkers. In addition, steroids themselves may have a therapeutic effect and could potentially confound results, as recent data has demonstrated a therapeutic effect in triple negative breast cancer [11]. Patients taking anti-retrovirals are also excluded since these medications are known to cause metabolic syndrome.

Chronic disease: Patients are ineligible if they have co-morbidities in which weight loss can result in adverse consequences such as kidney disease requiring dialysis, obstructive/restrictive lung disease requiring oxygen or heart failure requiring vasopressors or inotropes.

\section{Diet and behavioral modification}

CR has been defined differently in clinical trials but largely refers to a reduction of $20-40 \%$ or the patients' calories. Preclinical data suggests that a $30 \%$ caloric reduction could significantly impact tumor growth and metastases [2] when combined with radiation but this trial implemented a $25 \%$ reduction to make the regimen tolerable 
for patients and induce a similar positive stress that was induced in preclinical models. To determine the number of calories to be reduced per patient, baseline caloric intakes are determined by a 7-10 day 7-10 day food diary before their radiation treatment planning visit. This was offered as a diet journal in a notebook, or online. On the day of their radiation simulation/planning visit, patients meet with a dietary counselor to obtain guidelines for dietary modification. The total number of calories was determined for each day of the control period and a mean number of calories was determined for that 7-10 day period. A calculation for a $25 \%$ reduction was performed and the patient was given that reduced number of calories as a new baseline to achieve. During the dietary counseling visit, the target number of calories was discussed. Individualized recommendations made to suggest of simple substitutions of foods the patient is already eating and healthy eating choices are discussed. Patients begin their new diet the day after simulation and continue with the dietary intervention for two weeks prior to the start of radiation treatment. They continue CR through 6 weeks of standard fractionation radiation therapy, and then finish with an additional 2 weeks of $\mathrm{CR}$ after radiation therapy is completed.

We believe a few unique aspects of this clinical trial design have helped increase rates of adherence to the dietary intervention. A userfriendly online food diary (www.myfitnesspal.com), which also has an application for smart phones, is being used for the patients to report their caloric intake. The patients are encouraged to log their calories daily which helps with patient adherence because the total calorie count is immediately calculated to help the patient determine how close they are to their daily target. The convenience of the online food diary makes patients more likely to enter data correctly and to routinely log their meals and caloric intake. CaReFOR trial patients are provided with an access code to the website which is also given to our doctors and dietary counselors to monitor progress in real time. The patient is aware that this is regularly monitored, which may provide additional motivation. The patients also have a formal meeting with their physician and dietary counselor each week at which time they are weighed and current BMI is calculated. To determine accurate entry, patients are spot checked and questioned about their recall of their food consumption at weekly weigh-ins. A potential benefit of the CR diet in our trial is patients' ability to consume food of their own preference. Although this does not allow the investigator strict controls over macro and micronutrients consumed, and may be a source of variability, it does not detract from the goal of calorically restricting the patient.

Patient adherence is reinforced through positive cues. First, the rationale as to why the study is being performed is provided to each patient. When the trial is discussed with the patient, the potential benefits of a dietary intervention are explained. Although patients understand that this is a pilot trial investigating diet adherence, there are preclinical data suggesting that the efficacy of radiation is enhanced with caloric restriction and that the results of this trial could be used for future studies. It is also explained that more than half of breast cancer patients gain weight during cancer treatment and this is linked to worse clinical outcomes. In this manner, the tools learned during this pilot intervention may be used to motivate the patient to decrease the risk of recurrence in the future. Secondly, weekly meetings between the patient, physician and dietary counselor provide positive reinforcement as well. Diet logs are reviewed and any effective changes they have made in their diet are emphasized and positively discussed in the same manner as achieving target calorie and weight goals.

The last unique aspect we have implemented in the trial to help with adherence to the diet is the addition of behavioral modification plans.
One of the best defined and tested plans is the diabetes prevention program (DPP) [12]. For diabetic patients, it was demonstrated that sustained lifestyle interventions could prevent the pre-diabetic state from progressing to Type 2 Diabetes through behaviors to improve glucose tolerance. For the CaReFOR Trial, 10 of the DPP Lifestyle Balance Program Materials were adapted. Each lesson is reviewed with patients every week by our dietary counselors. Lessons adapted from the DPP for the CaReFOR Trial include how to read labels on food, how to cut down on fat intake, ways to best choose healthy options when eating out, and how to best deal with temptation. Each lesson is reviewed for approximately 20 minutes and is performed during weekly diet $\log$ review and weighing.

\section{Study endpoints}

Primary endpoint: Feasibility and adherence: The CaReFOR trial is the first clinical trial to combine CR with standard radiation therapy for patients actively undergoing definitive cancer treatment. It has been observed that standard fractionated radiation for early stage breast cancer patients can be difficult for patients to complete since it is delivered once daily over the course of 6 weeks ( 30 treatments), with some studies demonstrating an adherence rate of approximately $87 \%$ when patients are soley undergoing radiation therapy due to issues with toxicity and the daily commitment and travel for treatments [13]. The primary goal of the current study is to determine if patients can combine a dietary intervention during their radiation treatment with an acceptable adherence rate. For each of the 10 weeks of the intervention phase, an average daily caloric intake will be computed. If there are at least 4 diary logs during a particular week, and the resulting average daily caloric intake is less than the restricted target (75\% of the baseline daily average), that week will be considered adherent. If at least $80 \%$ of the intervention weeks (e.g., at least 8 of 10) are determined to meet this adherence definition, then the patient will be considered adherent for the study.

\section{Secondary endpoints}

Currently, there are no well-defined objective measures to monitor compliance to a dietary intervention or effectiveness. The secondary endpoints were therefore designed to determine if objective measures such as changes in body composition or serum metabolites might be used in the future to determine success of a dietary intervention.

Weight and body composition: Historically, weight loss has been shown to be an important surrogate marker of compliance to diet [14]. Patients on our protocol are weighed at weekly intervals at ontreatment visits and calculated BMI is recorded. Additionally, body fat measurements 4 point skinfold measurement and body composition (percent of body fat versus lean tissue) using a bioimpedance meter, are recorded at baseline, conclusion of diet and 4 weeks after diet is complete.

Translational endpoints serum and tissue: We have identified a key panel of molecules that may serve as potential markers of dietary adherence. Based on previously published literature regarding dietary intervention in humans [14] and current unpublished pre-clinical data in our laboratory, specific serum alterations have been demonstrated correlating with dietary changes. Fasting serum is drawn at baseline, at the conclusion of the diet and 4 weeks after the diet is complete. Standard laboratory tests include: hemoglobin A1C, triglycerides, erythrocyte sedimentation rate (ESR), adiponectin, leptin, estradiol and IGF-1. Specific research assays will be done and will include cytokine panels and miR-21 assessment. 
Toxicity evaluation: Skin toxicity is a known side effect of standard fractionated radiation. Although it is thought that dietary interventions such as CR or intermittent fasting may decrease side effects of cancer therapies such as chemotherapy or radiation therapy, this has not yet been proven in the setting of a clinical trial. A pilot study has shown that an extended fast right before chemotherapy, can decrease treatment induced neutropenia. Therefore, in this trial the Common Toxicity Criteria and Adverse Events (CTCAE version 4.0 scale22) is used to evaluate acute toxicity related to radiation such as skin side effects and fatigue. This is being compared to matched historical controls receiving the same dose and fractionation. Toxicity is charted through the entire course of radiation and the CTCAE score is documented weekly.

Psycho-social evaluation: It is well known that cancer treatment can cause a decline in a patient's emotional well-being and can induce fatigue and it is suspected that the positive stress of a dietary intervention will lessen the severity of these reactions typically noted with standard fractionation of radiation. The importance of patient reported outcomes in clinical trials is becoming increasingly more apparent. There can be discrepancies between patient reported toxicity and physician reported toxicity and it is therefore crucial that both be accounted for in clinical trial design. Typically, patient reported fatigue and emotional well-being during radiation therapy is typically wellmanaged. However, it is uncertain if a dietary intervention may cause worsening of these symptoms given the potential for added stress during treatment. Therefore, the patient's psycho-social status is evaluated with the FACT-B survey and the PROMIS cancer fatigue short form survey. These particular surveys are effective tools and are simple to measure and accurately quantify. FACT-B is the FACT (Functional Assessment of Cancer Therapy) questionnaire that has been extensively tested for breast cancer patients undergoing therapy and measures QOL based on Physical Well-Being, Social/Family Well-Being, Emotional Well-Being, and Functional Well-Being. The PROMIS short form is a generic disease-related survey that evaluates self-reported fatigue levels within the last 7 days. Its scores range from a mild lack of energy to an insurmountable tiredness that leads to a disruption of daily routine and social function. FACT-B and PROMIS questionnaires are administered at baseline, half way through the diet, at the end of the diet and then 4 weeks after the dietary intervention has completed.

\section{Statistical considerations}

Primary endpoint: We will compute the proportion of patients who are adherent to the CR protocol, along with a 95\% exact confidence interval. We will test whether individual patient compliance to 10 weeks of CR is greater than or equal to $80 \%$ through an exact binomial test (with one-sided alpha of 0.05).

Secondary endpoints: The change in body fat measurements between baseline and the end of the study will be analyzed paired $\mathrm{t}$-tests. Changes over time will be assessed by modeling these outcomes as a function of time (baseline, diet weeks $1,3,5,7$, and 10) mixedeffects regression. The patterns of change over time in vital signs, insulin and other clinical labs, serum markers, and psycho-social outcomes (FACT-B and PROMIS) will also be analyzed similarly. If some of these measures are skewed, appropriate transformations (e.g., $\log$ ) will be needed before the analyses. Finally, local recurrence, distant metastases, progression free survival, and overall survival will be analyzed survival methods, specifically the Kaplan-Meier method and the logrank test. If sufficient events occur, we may assess the impact of various patient and clinical/treatment variables on these outcomes Cox proportional hazards regression. Furthermore, local recurrence, distant metastases, progression free survival, and overall survival will be compared with historic controls.

\section{Sample size calculations}

The first phase of the trial was designed to enroll 40 women. If the primary objective is achieved, then the trial will enroll an additional 50 women (for a total of 90).

The trial's main objective is to establish that adherence to the CR protocol is sufficiently high (specifically, greater than $60 \%$ ) to allow the intervention to be used in future therapeutic trials. Thus, if the true adherence rate is $80 \%$, the trial will have $83 \%$ power after the first phase $(\mathrm{N}=40$, using an exact binomial test, with a one-sided alpha of 0.05$)$.

The trial's secondary endpoints will be assessed after the second phase $(\mathrm{N}=90)$. The trial is not formally powered for these endpoints, but it does have reasonable power to detect modest effects. For example, regarding the change between baseline and end-of-study BMI, power is about $83 \%$ for detecting an average change equal to about a third of the standard detion, i.e., $\sim 2.5 \mathrm{~kg} / \mathrm{m}^{2}$, assuming a standard detion of $8 \mathrm{~kg} /$ $\mathrm{m}^{2}$ (with $\mathrm{N}=90$, using a paired t-test, with a two-sided alpha of 0.05 ) [Figure 1 and 2].

\section{Discussion}

It is commonly believed that dietary alterations may not be an ideal therapeutic option for cancer patients due to concerns regarding weight loss and malnutrition. Dietary interventions have previously been

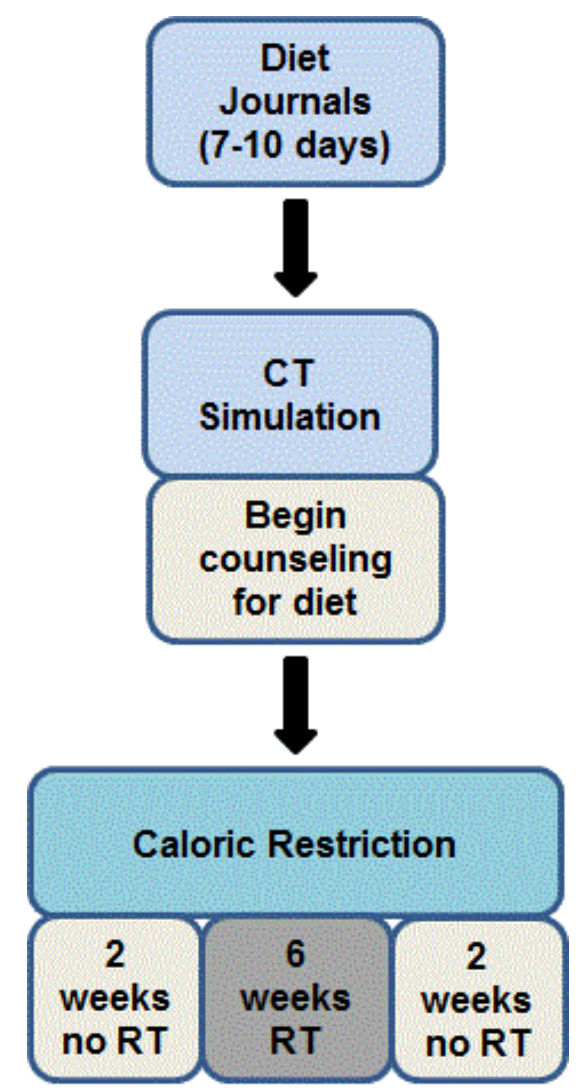

Figure 1. Algorithm for identification and enrollment of patients in the CaReFOR trial. Patients were considered eligible so long as they were to have lumpectomy for an early-stage breast cancer. All patients seen by our breast surgery team were screened for eligibility at weekly multidisciplinary tumor board. Initially, patients were excluded if they required adjuvant chemotherapy; however, to increase catchment, this was changed Patients were asked to keep a baseline diet log from which their calorie restriction was calculated. Diet was initiated after simulation visit and continued through 6 weeks of radiation therapy as well as two additional weeks following radiation. Lastly, patients had a follow-up visit 6 weeks after completion of radiation therapy. 


\section{Primary Outcomes}

Dietary Compliance: 24 -hour recall and diet logs reviewed $\checkmark$

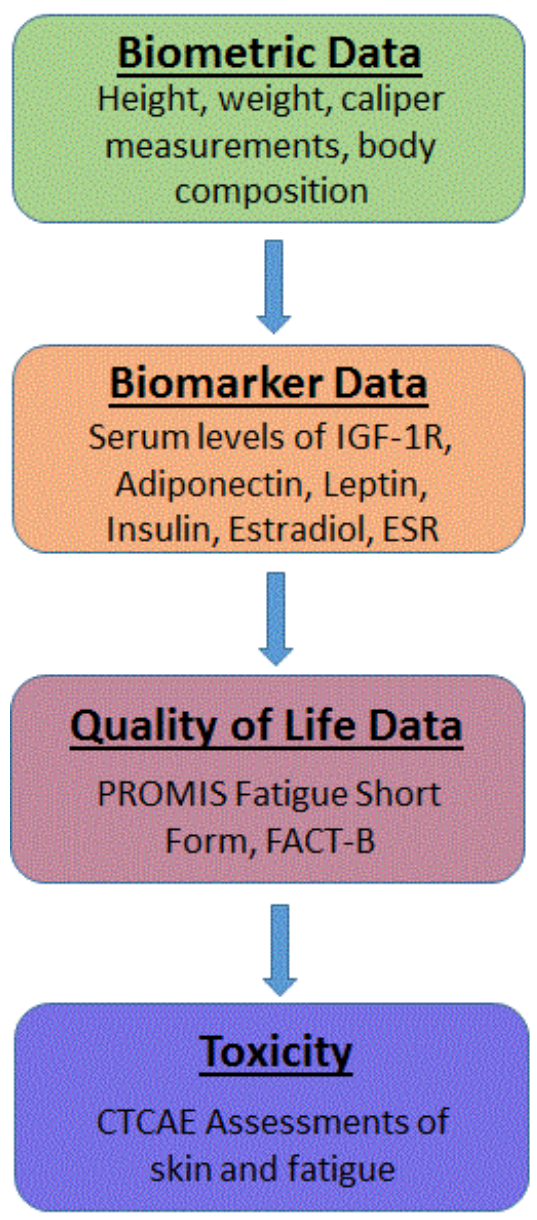

Figure 2. Schematic of primary and secondary outcome measurements in the CaReFOR trial. Feasibility of compliance to a dietary intervention was the primary outcome of this trial and therefore, patient diet logs were reviewed via an online collecting system. In addition, patients were asked to complete 24-hour recalls in person to assess accuracy of reporting. Secondary endpoints were biomarkers, biometrics and quality of life measures as pre-specified. These measurements were done at initial visit, immediately after completion of radiation and at the follow-up visit 6 weeks after completion of radiation.

used for cancer prevention and in the survivorship phases, but have not been administered in the setting of definitive treatment. Obstacles impeding implementation of dietary intervention include difficulties in administration, patient adherence, and objective monitoring. When designing a clinical trial using a dietary intervention, there are more variables that need to be considered compared with other interventions. However, given the potential benefits and lack of additional toxicity, it is a therapeutic option that should strongly be considered and defined.

Despite the belief that dietary interventions are beneficial by many practitioners, there has certainly been controversy when considering this as a therapeutic intervention. One of the main areas of concern lies with patient adherence and how this could be objectively monitored. Therefore, we have centered our initial trial design on the primary endpoint of successfully integrating dietary modification into standard radiation treatment. Through our secondary aims, we seek to determine if objective laboratory measures can more accurately determine diet adherence when compared to a dietary log.

Many patients in the radiation oncology clinic inquire about potential dietary interventions they may start which can help them during their treatment course. Since most oncology patients have an inherent interest in diet modification, we believe this intervention may be better tolerated and have higher adherence than anticipated. It is understood that a CR intervention requires a significant commitment from both the patient and the treating team. Our trial is unique in that it utilizes an instant feedback system for logging calorie intake, which allows patients to adapt better to the behavioral changes needed for the trial. It also promotes achievement of goals and holds patients accountable for their choices. We believe that consistent reinforcement during weekly weigh-ins and conversations with study staff are crucial to patient success. Constant feedback from study staff, including positive reinforcement of successes and reassurance in failures, has been essential to maintaining patient participation through 10 weeks of diet.

There are myriad of reasons why dietary interventions can be difficult for patients to adhere to; some of which are psychological. In these individuals, research shows that an inappropriate focus on physical benefits, and thus a lack of instant gratification from dieting, leads to diet discouragement [15]. In a study conducted by Caroline Ouwenhand, those who have success dieting are people who perform the appropriate healthy action when exposed to attractive foods, and those who fail at dieting are those who indulge in these foods after exposure [16]. In a study conducted by Papies, et al. titled "Goal Priming in Dieters: Recent Insights and Applications," psychological cues involved with dieters were evaluated. Unhealthy patient eating behaviors increase the difficulty of dieting, and exacerbate necessary behavioral modifications [17]. It is difficult for patients to change their eating behaviors when they have conditioned themselves to day-to-day cues that enable unhealthy eating. It has also been shown that after a day of compliant dieting, a patient may consider it a reward to sway from the diet on a subsequent day. Being in an environment with easy access to high-calorie, low-nutrient food also provides problems for dieters [17]. Papies establishes goal-priming as a method to diet success. If one is constantly reminded of dieting goals, he or she will more likely stick to the diet.

The design of our trial has several strategies in place to overcome the possible issues addressed above. Our focus on the benefits caloric restriction may have in breast cancer treatment gives patients greater incentive to follow the dietary protocol. In our trial, through constant patient contact and a daily diary requirement, patients are reminded of caloric intake and consumption goals. Through frequent input of diary entries, they are held accountable for adherence. Our patients have easy access to the online diet log that contains caloric and nutritional content for most common foods and meals.

A potential shortcoming of this study design is that this trial is reducing overall calories and is not addressing specific alterations in macro and micro nutrients. We believe that the primary therapeutic effect is achieved through total calorie reduction, while the effects of manipulating macronutrients are secondary. The goal is to determine if a straightforward dietary intervention itself is at all possible before 
defining specific parameters for future dietary trials. The potential for toxicity minimization and recurrence reduction, other clinical benefits of CR can be investigated in the future using a similar protocol and implementation system to ours. Future studies may reveal more suitable macronutrient parameters for metabolic intervention in specific cancers

Caloric restriction has been supported as a ble form of cancer treatment in the pre-clinical realm, but it is yet to be established as a standard intervention in the clinic. Although the process of dietary intervention is complicated, our protocol and the methods discussed may serve as the framework for future implementation in larger clinical trials. Participant compliance in this intervention model cannot be controlled, similar to other drug trials that rely on patients to self-administer medications. However, CR can be monitored and facilitated through careful planning, direct contact, a convenient logging system, and through patient effort to maintain transparency in what they consume. Currently, the CaReFOR trial remains the only clinical trial to combine CR concurrently with radiation therapy, but there are a number of newly opened trials using CR in combination with other cancer therapies including surgery and chemotherapy. The ultimate goal of this first-in-human CR trial during cancer therapy, is to establish patient compliance to $\mathrm{CR}$ and to expand the use of CR with our protocol into multi-institutional trials. Based on preclinical studies, it is anticipted that CR can provide benefit to cancer patients by both augmenting the effects of standard cancer therapeutics while mitigating toxicity. We believe that this protocol lays the foundation to accomplish these goals and expand the scope of using CR as a cancer therapy in different cancers with various stages and subtypes

\section{References}

1. Markowska A, Pawa Owska M, Filas V, Korski K, Grybo M, et al. (2013) Does Metformin affect ER, PR, IGF-1R, beta-catenin and PAX-2 expression in women with diabetes mellitus and endometrial cancer? Diabetol Metab Syndr 5: 76.

2. Saleh AD, Simone BA, Palazzo J, Savage JE, Sano Y, et al. (2013) Caloric restriction augments radiation efficacy in breast cancer. Cell Cycle 12: 1955-1963.

3. De Lorenzo MS, Baljinnyam E, Vatner DE, Abarzua P, Vatner SF, et al. (2011) Caloric restriction reduces growth of mammary tumors and metastases. Carcinogenesis 32: 1381-1387.
4. Ewertz M, Jensen MB, Gunnarsdóttir KÁ, Højris I, Jakobsen EH, et al. (2011) Effect of obesity on prognosis after early-stage breast cancer. J Clin Oncol 29: 25-31. [Crossref]

5. Goodwin PJ, Ennis M, Pritchard KI, McCready D, Koo J, et al. (1999) Adjuvant treatment and onset of menopause predict weight gain after breast cancer diagnosis. $J$ Clin Oncol 17: 120-129.

6. Kroenke CH, Chen WY, Rosner B, Holmes MD (2005) Weight, weight gain, and survival after breast cancer diagnosis. J Clin Oncol 23: 1370-1378. [Crossref]

7. Kroenke CH, Fung TT, Hu FB, Holmes MD (2005) Dietary patterns and survival after breast cancer diagnosis. J Clin Oncol 23: 9295-9303. [Crossref]

8. Imayama I, Alfano CM, Neuhouser ML, George SM, Wilder Smith A, et al. (2013) Weight, inflammation, cancer-related symptoms and healthrelated quality of life among breast cancer survivors. Breast Cancer Res Treat 140: 159-176.

9. McIver CM, Wycherley TP, Clifton PM (2012) MTOR signaling and ubiquitinproteosome gene expression in the preservation of fat free mass following high protein, calorie restricted weight loss. Nutr Metab (Lond) 9: 83. [Crossref]

10. Morley JE, Chahla E, Alkaade S (2010) Antiaging, longevity and calorie restriction. Curr Opin Clin Nutr Metab Care 13: 40-45. [Crossref]

11. Skor MN, Wonder EL, Kocherginsky M, Goyal A, Hall BA, et al. (2013) Glucocorticoid receptor antagonism as a novel therapy for triple-negative breast cancer. Clin Cancer Res 19: 6163-6172.

12. Diabetes Prevention Program Research G (2002) The Diabetes Prevention Program (DPP): description of lifestyle intervention. Diabetes Care 25: 2165-2171.

13. Srokowski TP1, Fang S, Duan Z, Buchholz TA, Hortobagyi GN, et al. (2008) Completion of adjuvant radiation therapy among women with breast cancer. Cancer 113: 22-29. [Crossref]

14. Alhassan S, Kim S, Bersamin A, King AC, Gardner CD (2008) Dietary adherence and weight loss success among overweight women: results from the A TO Z weight loss study. Int J Obes (Lond) 32: 985-991. [Crossref]

15. Leske S, Strodl E, Hou XY (2012) A qualitative study of the determinants of dieting and non-dieting approaches in overweight/obese Australian adults. BMC Public Health 12: 1086.

16. Ouwehand C1, Papies EK (2010) Eat it or beat it. The differential effects of food temptations on overweight and normal-weight restrained eaters. Appetite 55: 56-60. [Crossref]

17. Papies EK (2012) Goal Priming in Dieters: Recent Insights and Applications. Curr Obes Rep 1: 99-105. [Crossref]

Copyright: (C2017 Simone BA. This is an open-access article distributed under the terms of the Creative Commons Attribution License, which permits unrestricted use, distribution, and reproduction in any medium, provided the original author and source are credited. 